Einführung zum Thema

Unfallchirurg $2021 \cdot 124: 431-432$

https://doi.org/10.1007/s00113-021-01003-9

Angenommen: 7. April 2021

(c) Springer Medizin Verlag GmbH, ein Teil von Springer Nature 2021

\author{
W. Mutschler $\cdot$ H. Locher ${ }^{2,3}$ \\ 'Klinik für Allgemeine, Unfall- und Wiederherstellungschirurgie, LMU Klinikum, München, Deutschland \\ ${ }^{2}$ Zentrum für Orthopädie und Unfallchirurgie, Tettnang, Deutschland \\ ${ }^{3}$ Klinikum rechts der Isar, TU München, München, Deutschland
}

\title{
Funktionelle Therapieverfahren in der Unfallchirurgie
}

\section{Manuelle Medizin, Osteopathie, Physiotherapie und Ergotherapie}

die Betrachtungsebenen Zelle und intrazelluläre Vorgänge, Gewebeebene, Organebene und Körperebene miteinander verknüpft werden und in ihren Wechselwirkungen verstanden werden. Schmerz umfassend zu begreifen, ist beispielsweise eine echte intellektuelle Herausforderung.

Dabei sollte man wenigstens die Prinzipien und Methoden der manuellen $\mathrm{Me}$ dizin, Osteopathie, physikalischen Therapie und Ergotherapie i. Allg. verstehen, denn letztlich stellen wir Ärzte die Indikation dafür, verordnen sie und sollen den Heilungsverlauf steuern und kontrollieren. Wie soll das ohne Verständnis gehen?

Und ein weiteres. Selbst wenn Sie selbst manuelle Medizin und Osteopathie kritisch beurteilen, ihre Patienten werden das anders sehen. Die entsprechenden Praxen in Deutschland sind voll von Rat- und Hilfesuchenden und finden in der großen Mehrheit Hilfe bei im wahrsten Sinne des Wortes handwerklich geübten Ärzten und Therapeut*innen. Warum dann nicht der vielbeschworene Anwalt des Patienten bleiben und ihn fach- und sachkundig beraten? Der Eindruck einer Parallelwelt etwa zwischen $\mathrm{O} \& \mathrm{U}$ und Osteopathie mag zugespitzt erscheinen, aber wenn es so wäre, sind wir daran nicht ganz unschuldig.

Daraus leitet sich unser Anliegen für das Themenheft ab: Ihnen diese wichtigen Bereiche der konservativen Therapie zusammengefasst und aufeinander bezogen darzustellen.
Uns war es in der Umsetzung dann wichtig, Autor*innen zu finden, die in den genannten vier therapeutischen Feldern selbst tätig sind, ihre immense praktische Erfahrung anschaulich schildern können und uns gleichzeitig die zugrunde liegenden wissenschaftlichen Theorien und Theoreme nahebringen können. Um dabei deren Arbeitsweise so konkret wie möglich kennenzulernen, haben wir uns darauf verständigt, jeweils Fallbeispiele aus dem Bereich des verletzten distalen Handgelenks und der Hand auszuwählen. In der Zusammenschau sollte sich unseren Leser*innen dann erschließen, welche verschiedenen Methoden sinnvollerweise zum Einsatz kommen können, um das gemeinsame Behandlungsziel einer schmerzfreien vollen Funktion und beruflichen und sozialen Reintegration zu erreichen. Alle Autor*innen haben dem zugestimmt und ihre Beiträge vorbildlich aufeinander abgestimmt.

Wovon handeln die vier Artikel des Themenheftes im Einzelnen?

Manuelle Medizin ist die medizinische Disziplin, die sich umfassend mit der Diagnose, Therapie und Prävention reversibler Funktionsstörungen am Bewegungsorgan und anderen damit verbundenen Organsystemen befasst. Prof. Dr. Hermann Locher ist als niedergelassener Facharzt für Orthopädie und Unfallchirurgie in Tettnang tätig, kommt einem akademischen Lehrauftrag für manuelle Medizin und Schmerztherapie an der TU München nach und ist in einer Reihe berufspolitischer Gremien und wissen- senschaftliche Grundlage zu erarbeiten, ist extrem komplex; schließlich müssen 
schaftlicher Vereinigungen für manuelle Medizin engagiert. Er hat in seinem Beitrag die Quintessenz aus dem von ihm mitherausgegebenen Lehrbuch Manuelle Medizin zusammengefasst. Anhand einprägsamer schematischer Abbildungen stellt er zunächst die neuroanatomischen und neurophysiologischen Grundlagen der Wirkungsweise der manuellen Medizin anschaulich dar.

\section{》) Der Schlüssel liegt im Verständnis des Schmerzes als komplexes Geschehen}

Der Schlüssel liegt im Verständnis des Schmerzes als komplexes Zusammenwirken afferenter und efferenter Bahnen vorwiegend auf Rückenmarkebene mit weitergehender Verschaltung im Gehirn. Die Hände des Therapeuten greifen in diese reflektorischen Regelkreise v.a. durch die Beeinflussung schmerzinhibitorischer Systeme ein.

Anschließend führt er in die Begrifflichkeiten der Osteopathie ein. Osteopathie wird heute als Bestandteil und Erweiterung der manuellen Medizin gesehen. Sie hat aber immer noch gegen ihren Ursprung in der Naturheilkunde und Komplementärmedizin zu kämpfen und auch damit, dass osteopathische Diagnostik und Therapie von verschiedenen Berufsgruppen wie Ärzten, Physiotherapeuten, Masseuren und Heilpraktikern ausgeübt werden und die Qualifikationen und Anwendungsstandards dementsprechend sehr heterogen sind. Eine Standortbestimmung zu "good clinical practice" in der Osteopathie erschien daher geboten. Nach der Lektüre des Artikels sollte klar sein, dass bei Einhaltung dieser Standards der Osteopathie solides Handwerk zugrunde liegt und die manuelle Medizin belastbare wissenschaftliche Literatur vorlegt. Dies wird am Beispiel der evidenzbasierten Wirksamkeit manualtherapeutischer Maßnahmen bei häufigen Krankheitsbildern wie Lumbalgie, zervikogener Kopfschmerz oder NackenSchulter-Arm-Syndrom gezeigt.

Frau Magga Corts leitet eine Praxis für Osteopathie und Naturheilkunde in Köln und ist als Verfasserin mehrerer Bücher zur Osteopathie ausgewiesen. In ihrem Artikel legt sie den Schwerpunkt auf osteopathische Behandlungsmethoden in der postoperativen Nachbehandlung und wählt dazu das Beispiel einer Patientin mit distaler Radiusfraktur, anhand derer sie die spezifische osteopathische Ganzkörperdiagnostik hinsichtlich möglicher postoperativer Dysfunktionen und Schmerzen und gängige Behandlungstechniken mit illustrativen Bildern dazu schildert. Zuvor gibt sie in Ergänzung des Artikels von Prof. Locher einen ausführlichen Einblick in die Denkweise der Osteopathie, deren Kenntnis für eine vorurteilsfreie interdisziplinäre $\mathrm{Zu}$ sammenarbeit nur von Vorteil sein kann. Sie schließt mit der Aufforderung, osteopathische und physiotherapeutische Maßnahmen unbedingt aufeinander abzustimmen.

Deshalb kommt im nächsten Beitrag Frau Cornelia Schubert zu Wort, die wir über Prof. Pennig (Köln) gewinnen konnten und die die Kontakte zu den beiden anderen Autorinnen herstellte. Sie ist die Leitende Physiotherapeutin am St. Vinzenz Hospital in Köln und widmet sich dem heute dank präemptiver analgetischer Intervention eher seltenen, aber unverändert schwierig zu therapierenden Krankheitsbild des komplexen regionalen Schmerzsyndroms (CRPS) im Handbereich. Auch hier lohnt es sich, genau zu lesen und hinzuschauen, denn wo sonst wird aus erster Hand demonstriert, wie ein stadienorientierter Behandlungsablauf des CRPS beispielhaft praktisch gestaltet werden kann.

Frau Johanna Ismaier führt eine Praxis für Ergotherapie in Hohenbrunn bei München und hat ein Master-Studium in Prävention und Gesundheitspsychologie abgeschlossen. Umfassendes Ziel der Ergotherapie ist eine zufriedenstellende Ausführung alltäglicher Handlungen, indem beeinträchtigte Fähigkeiten und Funktionen verbessert, wiederhergestellt oder kompensiert werden. Überschneidungen bestehen dabei $\mathrm{zu}$ Beschäftigungstherapie, Arbeitstherapie und Physiotherapie. Ergotherapie hat als ganzheitlicher Ansatz ihren Platz in vielen Gebieten der Medizin, von der Pädiatrie bis zur Geriatrie. Frau Ismaier stellt hier die Tätigkeitsfelder der Ergotherapie wie Funktions- und Wahr- nehmungstraining, Hilfsmitteleinsatz, Ergonomieberatung, berufliche Rehabilitation und Belastungserprobung im Kontext zu O\&U dar und schildert pars pro toto die Ergotherapie bei komplexen Handverletzungen am Fall einer schweren Daumenverletzung.

Wir bedanken uns sehr herzlich bei allen Autorinnen für ihre instruktiven Beiträge und die gelebte Interdisziplinarität bei der Abstimmung der Beiträge. Bei so viel Handanlegen gewinnt das Bild vom „Hand-in-Hand-Arbeiten“ eine mehrschichtige Bedeutung.

Wir hoffen, dass Sie sich im Folgenden auf dieses ungewohnte Terrain einlassen und ihr Wissen über die manuelle Medizin, Osteopathie, Physiotherapie und Ergotherapie gerne erweitern und vertiefen. Dies gilt im Besonderen für alle Kolleg*innen, die sich in der Facharztweiterbildung befinden, denn in der neuen Muster-Weiterbildungsordnung von 2018 sind Kenntnisse in den Grundlagen manualmedizinischer Verfahren und in den Grundlagen alternativer Heilmethoden nachzuweisen.

Prof. Dr. W. Mutschler

Prof. Dr. H. Locher

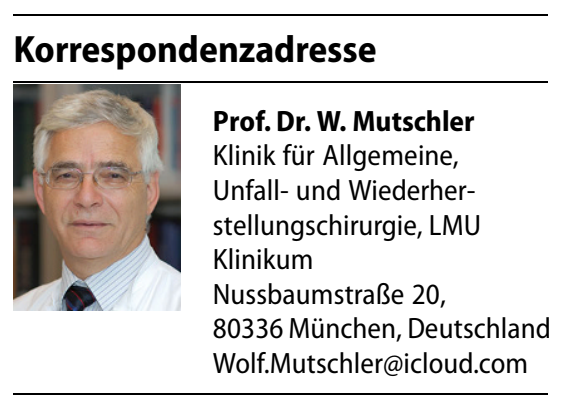

Interessenkonflikt. W. Mutschler und H. Locher geben an, dass kein Interessenkonflikt besteht. 